\title{
On Shioda's problem about Jacobi sums
}

\author{
by \\ Hiroo Miki (Bures-sur-Yvette and Kyoto)
}

In the present paper, we will give a positive result relating to the $l$-part of Shioda's problem [2] on Jacobi sums $J_{l}^{(a)}(\mathfrak{p})$ under a certain condition (see Corollary to Theorem 2 of the present paper), as an application of our congruence for Jacobi sums [1, Theorem 2] (see also Theorem 1 of the present paper).

Let $l$ be any prime number such that $l \geq 5$, and let $\zeta_{l}$ be a primitive lth root of unity in $\mathbb{C}$ (the field of complex numbers). Let $\mathbb{Q}$ be the field of rational numbers and let $\mathbb{Z}$ be the ring of rational integers. Put $k=\mathbb{Q}\left(\zeta_{l}\right)$. For any integer $r \geq 1$ and any $a=\left(a_{1}, \ldots, a_{r}\right) \in \mathbb{Z}^{r}$ and for any prime ideal $\mathfrak{p}$ of $k$ which is prime to $l$, let

$$
J_{l}^{(a)}(\mathfrak{p})=(-1)^{r+1} \sum_{\substack{x_{1}, \ldots, x_{r} \in \mathbb{F}_{q} \\ x_{1}+\ldots+x_{r}=-1}} \chi_{\mathfrak{p}}^{a_{1}}\left(x_{1}\right) \ldots \chi_{\mathfrak{p}}^{a_{r}}\left(x_{r}\right) \in \mathbb{Z}\left[\zeta_{l}\right],
$$

be the Jacobi sum, where $\mathbb{F}_{q}=\mathbb{Z}\left[\zeta_{l}\right] / \mathfrak{p}, q=N \mathfrak{p}=\#\left(\mathbb{F}_{q}\right)$, and $\chi_{\mathfrak{p}}(x)=\left(\frac{x}{\mathfrak{p}}\right)_{l}$ is the $l$ th power residue symbol in $k$, i.e., $\chi_{\mathfrak{p}}(x \bmod \mathfrak{p})$ is a unique $l$ th root of unity in $\mathbb{C}$ such that

$$
\chi_{\mathfrak{p}}(x \bmod \mathfrak{p}) \equiv x^{(N \mathfrak{p}-1) / l}(\bmod \mathfrak{p})
$$

for $x \in \mathbb{Z}\left[\zeta_{l}\right], x \notin \mathfrak{p}$, and $\chi_{\mathfrak{p}}(0)=0$.

If $r \geq 3$ is odd and if $a_{i} \not \equiv 0(\bmod l)$ for all $i(0 \leq i \leq r)$ (with $\left.a_{0}=-\sum_{i=1}^{r} a_{i}\right)$, then by Shioda [2, Corollary 3.3] we can write

$$
N_{k / \mathbb{Q}}\left(1-J_{l}^{(a)}(\mathfrak{p}) q^{-(r-1) / 2}\right)=B l^{3} / q^{w},
$$

where $N_{k / \mathbb{Q}}$ is the norm mapping from $k$ to $\mathbb{Q}, B$ and $w$ are non-negative integers, and $w$ is defined by (2.8) of [2].

Shioda's PRoblem (see [2, Question 3.4]). Is $B$ a square if $B \neq 0$ ?

Zagier [4] (see [2, Example 3.5] and [3, Examples 5.15.1]) verified it by computer in the case where $l<20$ and $p<500, p \equiv 1(\bmod l)$, where $p$ is a prime number in $\mathfrak{p}$. Shioda $[2$, Theorem 7.1$]$ proved that $B$ is a square, 
possibly multiplied by a divisor of $2 l p$ when $r=3$, and Suwa and Yui [3, Corollary 5.14.1] proved that $B$ is divisible by $p$ exactly even times under a certain condition when $r=3$.

Let $\overline{\mathbb{Q}}$ be the algebraic closure of $\mathbb{Q}$ in $\mathbb{C}$ and let $\overline{\mathbb{Q}}_{l}$ be a fixed algebraic closure of the field of $l$-adic numbers $\mathbb{Q}_{l}$. By means of a fixed imbedding $\overline{\mathbb{Q}} \hookrightarrow \overline{\mathbb{Q}}_{l}$, we consider $\overline{\mathbb{Q}}$ as a subfield of $\overline{\mathbb{Q}}_{l}$. We also consider that all algebraic extensions of $\mathbb{Q}_{l}$ and all elements which are algebraic over $\mathbb{Q}_{l}$ are contained in $\overline{\mathbb{Q}}_{l}$. All congruences in the present paper are those in $\overline{\mathbb{Q}}_{l}$.

For any odd $m(3 \leq m \leq l-2)$, put

$$
E_{m}=\prod_{d=1}^{l-1}\left(1-\zeta_{l}^{d}\right)^{m_{d}}
$$

where $m_{d} \in \mathbb{Z}$ is such that $m_{d} \equiv d^{m-1}(\bmod l)$ and $\sum_{d=1}^{l-1} m_{d}=0$. Let $\beta_{m}(\mathfrak{p}) \in \mathbb{Z}$ be such that

$$
\left(\frac{E_{m}}{\mathfrak{p}}\right)_{l}=\zeta_{l}^{\beta_{m}(\mathfrak{p})}
$$

Then $\beta_{m}(\mathfrak{p})$ is uniquely determined $\bmod l$ by $l, m$, and $\mathfrak{p}$.

Theorem $1\left(\left[1\right.\right.$, Theorem 2]). If $a=\left(a_{1}, \ldots, a_{r}\right) \not \equiv(0, \ldots, 0)(\bmod l)$, then

$$
\begin{aligned}
J_{l}^{(a)}(\mathfrak{p}) \equiv & N \mathfrak{p}^{-1} \cdot \operatorname{Exp}\left\{\sum_{\substack{3 \leq m \leq l-2 \\
m \text { odd }}}\left(\sum_{j=0}^{r} a_{j}^{m}\right) \beta_{m}(\mathfrak{p}) \frac{\pi^{m}}{m !}\right. \\
& \left.-\frac{N \mathfrak{p}-1}{2 l}\left(\sum_{j=0}^{r} a_{j}^{l-1}\right) \pi^{l-1}\right\}\left(\bmod \pi^{l}\right),
\end{aligned}
$$

where $a_{0}=-\sum_{j=1}^{r} a_{j}, \pi$ is a prime element of $\mathbb{Q}_{l}\left(\zeta_{l}\right)$ such that

$$
\pi \equiv \log \zeta_{l}\left(\bmod \left(\zeta_{l}-1\right)^{l}\right) \equiv \sum_{i=1}^{l-1}(-1)^{i-1}\left(\zeta_{l}-1\right)^{i} / i\left(\bmod \left(\zeta_{l}-1\right)^{l}\right)
$$

and

$$
\operatorname{Exp} X=\sum_{i=0}^{l-1} \frac{X^{i}}{i !} \in \mathbb{Z}_{l}[X]
$$

Remark. The sign of the coefficient of $\pi^{l-1}$ in the above formula is different from that of $[1$, Theorem 2], which was incorrect. 
LeMma 1. For any odd $m(3 \leq m \leq l-2)$,

$$
\begin{aligned}
E_{m} & \equiv d_{m} \operatorname{Exp}\left(-\frac{B_{j}}{j} \cdot \frac{\pi^{j}}{j !}\right)\left(\bmod \pi^{l-1}\right) \\
& \equiv d_{m}\left(1-\frac{B_{j}}{j} \cdot \frac{\pi^{j}}{j !}\right)\left(\bmod \pi^{j+1}\right),
\end{aligned}
$$

where $d_{m}=\prod_{d=1}^{l-1}(-d)^{m_{d}} \in \mathbb{Z}_{l}^{\times}$(the group of units in $\left.\mathbb{Z}_{l}\right), j=l-m$, and $B_{j}$ is the $j$-th Bernoulli number.

Proof. By definition,

$$
E_{m}=d_{m} \prod_{d=1}^{l-1}\left(\frac{1-\zeta_{l}^{d}}{-d \pi}\right)^{m_{d}} \quad \text { and } \quad \zeta_{l} \equiv \operatorname{Exp} \pi\left(\bmod \pi^{l}\right) .
$$

Easy computation shows that

$$
\log \frac{1-e^{t}}{-t}=\frac{1}{2} t+\sum_{i=2}^{\infty} \frac{B_{i}}{i} \cdot \frac{t^{i}}{i !}
$$

Hence

$$
\log \left(\frac{1-\zeta_{l}}{-\pi}\right) \equiv \frac{1}{2} \pi+\sum_{i=2}^{l-1} \frac{B_{i}}{i} \cdot \frac{\pi^{i}}{i !}\left(\bmod \pi^{l-1}\right),
$$

so

$$
\eta \log \left(\frac{1-\zeta_{l}}{-\pi}\right) \equiv-\frac{B_{j}}{j} \cdot \frac{\pi^{j}}{j !}\left(\bmod \pi^{l-1}\right),
$$

where $\eta=\sum_{d=1}^{l-1} m_{d} \sigma_{d} \in \mathbb{Z}_{l}\left[\operatorname{Gal}\left(\mathbb{Q}_{l}\left(\zeta_{l}\right) / \mathbb{Q}_{l}\right)\right]$ (the group ring of the Galois group $\operatorname{Gal}\left(\mathbb{Q}_{l}\left(\zeta_{l}\right) / \mathbb{Q}_{l}\right)$ over $\left.\mathbb{Z}_{l}\right)$ and $\sigma_{d} \in \operatorname{Gal}\left(\mathbb{Q}_{l}\left(\zeta_{l}\right) / \mathbb{Q}_{l}\right)$ is such that $\zeta_{l}^{\sigma_{d}}$ $=\zeta_{l}^{d}$, since

$$
\eta \pi^{i} \equiv \begin{cases}0\left(\bmod \pi^{l}\right) & \text { if } i \neq j \\ -\pi^{i}\left(\bmod \pi^{l}\right) & \text { if } i=j\end{cases}
$$

for $1 \leq i \leq l-1$. Hence

$$
\begin{aligned}
E_{m} & \equiv d_{m}\left(\frac{1-\zeta_{l}}{-\pi}\right)^{\eta}\left(\bmod \pi^{l-1}\right) \\
& \equiv d_{m} \operatorname{Exp}\left(-\frac{B_{j}}{j} \cdot \frac{\pi^{j}}{j !}\right)\left(\bmod \pi^{l-1}\right) .
\end{aligned}
$$

This completes the proof.

Put $K=k\left(\sqrt[l]{E_{m}} \mid m\right.$ odd, $\left.3 \leq m \leq l-2\right)$. We have $K \neq k$, since $B_{2}=\frac{1}{6} \in \mathbb{Z}_{l}^{\times}$implies $E_{l-2} \notin k^{l}$ by Lemma 1 . Since $E_{m}$ is a unit of $k, K / k$ is a finite abelian extension which is unramified outside $l$. 
By Theorem 1 we have directly the following

TheOREM 2. Let $\sigma=(\mathfrak{p}, K / k)$ denote the Frobenius automorphism of $\mathfrak{p}$ with respect to $K / k$. Assume $\sigma \neq 1$. Then

$$
J_{l}^{(a)}(\mathfrak{p}) \equiv 1+\left(\sum_{j=0}^{r} a_{j}^{m}\right) \beta_{m}(\mathfrak{p}) \frac{\pi^{m}}{m !}\left(\bmod \pi^{m+1}\right)
$$

and

$$
\beta_{m}(\mathfrak{p}) \not \equiv 0(\bmod l),
$$

where $m$ is the least odd $m(3 \leq m \leq l-2)$ such that $\left(\sqrt[l]{E_{m}}\right)^{\sigma} \neq \sqrt[l]{E_{m}}$.

COROLlaRY. Let the notation and assumptions be as in Theorem 2 and let $B$ be as in Shioda's problem. Furthermore, assume that $\sum_{j=0}^{r} a_{j}^{m} \not \equiv 0$ $(\bmod l)$. Then $\operatorname{ord}_{l}(B)=m-3$. In particular, $\operatorname{ord}_{l}(B)$ is even, where $\operatorname{ord}_{l}$ is the normalized additive valuation of $\mathbb{Q}_{l}$.

The above corollary gives an affirmative answer to the $l$-part of Shioda's problem when $(\mathfrak{p}, K / k) \neq 1$ and $\sum_{j=0}^{r} a_{j}^{m} \not \equiv 0(\bmod l)$.

Lemma 2. Let $K$ be as just before Theorem 2. Then $K$ and $k\left(\sqrt[l]{\zeta_{l}}\right)$ are linearly disjoint over $k$.

Proof. By Lemma 1,

$$
E_{m} \equiv d_{m}\left(\bmod \pi^{2}\right)
$$

If the assertion is false, then $k\left(\sqrt[l]{\zeta_{l}}\right) \subset K$, so by Kummer theory we can write

$$
\zeta_{l}=\prod_{\substack{3 \leq m \leq l-2 \\ m \text { odd }}} E_{m}^{\lambda_{m}} \cdot A^{l}
$$

with some $\lambda_{m} \in \mathbb{Z}$ and some $A \in k^{\times}$. Since $\zeta_{l}$ and $E_{m}$ are units of $k, A \equiv u$ $(\bmod \pi)$ with some $u \in \mathbb{Z}_{l}^{\times}$, so

$$
A^{l} \equiv u^{l}\left(\bmod \pi^{l}\right) .
$$

By (1)-(3),

$$
1+\pi \equiv b\left(\bmod \pi^{2}\right),
$$

where $b=\prod d_{m}^{\lambda_{m}} \cdot u^{l} \in \mathbb{Z}_{l}^{\times}$. Hence $b \equiv 1(\bmod \pi)$, so $b \equiv 1\left(\bmod \pi^{l-1}\right)$, since $b \in \mathbb{Z}_{l}$. This contradicts (4) and completes the proof.

Put $L=K\left(\sqrt[l]{\zeta_{l}}\right)=K\left(\zeta_{l^{2}}\right)$, where $\zeta_{l^{2}}$ is a primitive $l^{2}$ th root of unity. Then $L / k$ is a finite abelian extension of $k$ which is unramified outside $l$. The next theorem and its corollary give a partial result toward Shioda's problem when $\sigma \mid K=1$. 
Theorem 3. Put $\sigma=(\mathfrak{p}, L / k)$. Assume that $\sigma \mid K=1$ and $\zeta_{l^{2}}^{\sigma} \neq \zeta_{l^{2}}$. Then

$$
\begin{aligned}
J_{l}^{(a)}(\mathfrak{p}) & \equiv 1-\left(1-\frac{r^{\prime}}{2}\right)(q-1)\left(\bmod \pi^{l}\right) \\
& \equiv 1-\left(1-\frac{r^{\prime}}{2}\right) \lambda l\left(\bmod \pi^{l}\right)
\end{aligned}
$$

and $\lambda \not \equiv 0(\bmod l)$, where $\lambda=(q-1) / l \in \mathbb{Z}$ and $r^{\prime}=\#\left\{0 \leq i \leq r \mid a_{i} \not \equiv 0\right.$ $(\bmod l)\}$.

Remark. By Lemma 2 and Chebotarev's density theorem, there exist infinitely many prime ideals $\mathfrak{p}$ of $k$ of degree 1 satisfying the condition in Theorem 3.

Proof of Theorem 3 . The condition $\zeta_{l^{2}}^{\sigma} \neq \zeta_{l^{2}}$ is equivalent to $\lambda \not \equiv 0$ $(\bmod l)$, and the condition $\sigma \mid K=1$ is equivalent to $\beta_{m}(\mathfrak{p}) \equiv 0(\bmod l)$ for all odd $m(3 \leq m \leq l-2)$. Hence by Theorem 1 ,

$$
\begin{aligned}
J_{l}^{(a)}(\mathfrak{p}) & \equiv q^{-1}\left(1-\frac{q-1}{l} \cdot \frac{r^{\prime}}{2} \pi^{l-1}\right)\left(\bmod \pi^{l}\right) \\
& \equiv(1-\lambda l)\left(1+\lambda \cdot \frac{r^{\prime}}{2} \cdot l\right)\left(\bmod \pi^{l}\right) \\
& \equiv 1-\left(1-\frac{r^{\prime}}{2}\right) \lambda l\left(\bmod \pi^{l}\right) \\
& \equiv 1-\left(1-\frac{r^{\prime}}{2}\right)(q-1)\left(\bmod \pi^{l}\right),
\end{aligned}
$$

since $\pi^{l-1} \equiv-l\left(\bmod \pi^{l}\right)$. This completes the proof.

Corollary. Assume that $r \geq 3$ is odd and that $a_{i} \not \equiv 0(\bmod l)$ for all $i(0 \leq i \leq r)$. Let $\mathfrak{p}$ satisfy the condition in Theorem 3 . Put

$$
S=1-J_{l}^{(a)}(\mathfrak{p}) q^{-(r-1) / 2} .
$$

Then $S \equiv 0\left(\bmod \pi^{l}\right)$. In particular, $\operatorname{ord}_{l}\left(N_{k / \mathbb{Q}}(S)\right) \geq l$.

Proof. By Theorem 3,

$$
\begin{aligned}
J_{l}^{(a)}(\mathfrak{p}) q^{-(r-1) / 2} & \equiv\left(1-\left(1-\frac{r^{\prime}}{2}\right) \lambda l\right)\left(1-\frac{r-1}{2} \lambda l\right)\left(\bmod \pi^{l}\right) \\
& \equiv 1-\frac{1}{2}\left(r-r^{\prime}+1\right) \lambda l\left(\bmod \pi^{l}\right) .
\end{aligned}
$$

Hence $S \equiv \frac{1}{2}\left(r-r^{\prime}+1\right) \lambda l\left(\bmod \pi^{l}\right)$. Since $r^{\prime}=r+1$ by assumption, this gives the assertion.

Re mark. When $(\mathfrak{p}, L / k)=1$, Shioda's problem is still an open problem. 
This paper has been written during my stay at the I.H.E.S. in 1991/92. I would like to thank the Institute for their hospitality and its financial support. I would also like to thank Professors Don Zagier, Yuji Kida, and Masanobu Kaneko for supplying me further numerical data on Shioda's problem.

\section{References}

[1] H. Miki, On the l-adic expansion of certain Gauss sums and its applications, Adv. Stud. Pure Math. 12 (1987), 87-118.

[2] T. Shioda, Some observations on Jacobi sums, ibid. 119-135.

[3] N. Suw a and N. Yui, Arithmetic of certain algebraic surfaces over finite fields, in: Lecture Notes in Math. 1383, Springer, Berlin, 1989, 186-256.

[4] D. Zagier, Numerical data, March 1983 (see [3], Examples 5.15.1).

INSTITUT DES HAUTES ÉTUDES SCIENTIFIQUES

91440 BURES-SUR-YVETTE, FRANCE

DEPARTMENT OF LIBERAL ARTS AND SCIENCES

FACULTY OF ENGINEERING AND DESIGN

KYOTO INSTITUTE OF TECHNOLOGY

SAKYO-KU, KYOTO 606, JAPAN 